Case Report

\title{
Brucellosis Presenting with Pericarditis: Case Report and Literature Review
}

\author{
Fábio Lopes Pedro, ${ }^{1,2}$ Fernanda Paula Franchini, ${ }^{2}$ and Leonardo Muraro Wildner ${ }^{3}$ \\ ${ }^{1}$ Universidade Federal do Rio Grande do Sul, Porto Alegre, RS, Brazil \\ ${ }^{2}$ Hospital Universitário de Santa Maria, Santa Maria, RS, Brazil \\ ${ }^{3}$ Universidade Federal de Santa Maria, Santa Maria, RS, Brazil \\ Correspondence should be addressed to Fábio Lopes Pedro; fabiopedro.balboa@gmail.com
}

Received 19 December 2012; Accepted 10 January 2013

Academic Editors: D. L. Palazzi, A. C. Sena, and T. Shibata

Copyright (C) 2013 Fábio Lopes Pedro et al. This is an open access article distributed under the Creative Commons Attribution License, which permits unrestricted use, distribution, and reproduction in any medium, provided the original work is properly cited.

Pericarditis is a rare manifestation during the course of brucellosis. This paper describes a case of pericarditis associated with brucellosis in a 31-year-old veterinary physician with a past medical history of testicular tumor and reviews the cases of pericarditis associated with brucellosis in medical English literature.

\section{Introduction}

Brucellosis is a zoonotic disease caused by Gram-negative bacteria from the genus Brucella. With a worldwide distribution $[1,2]$, it is considered hyperendemic in South America [3]. More than half a million new cases are diagnosed each year [1], and the prevalence rate exceeds 10 cases per 100,000 inhabitants in some countries [2].

The infection is transmitted to humans by animals, due to occupational exposure or ingestion of contaminated dairy products and meats [1]. With a wide spectrum of clinical manifestations [4], it can affect any organ or tissue in the human body [1]. The most commonly affected systems are the musculoskeletal, gastrointestinal, genitourinary, hematological, and respiratory systems [5].

Acute cases typically present with chills, fever, asthenia, sweating, weight loss, and back pain [5]. The most common findings on physical examination are hepatomegaly and splenomegaly [6]. Cardiovascular complications are rare, [2] with endocarditis being the most common form of cardiovascular system involvement [7].

The purpose of this paper is to report a case of brucellosis that presented with mild pericarditis during its course, not associated with endocarditis or myocarditis.

\section{Case Report}

Male patient, Caucasian, 31 years old, from São Martinho da Serra, RS, Brazil. He resides with his wife and his mother. He had a past medical history of testicular tumor at age 24 and was cured after treatment. He had been asymptomatic for five years.

On $07 / 01 / 2012$, he started having fever $\left(40.2^{\circ} \mathrm{C}\right) . \mathrm{He}$ sought medical treatment with the assistant oncologist, who requested additional tests to rule out tumor recurrence. A total abdominal ultrasonography showed hepatosplenomegaly and retroperitoneal lymph node enlargement. A transthoracic echocardiogram showed signs suggestive of acute pericarditis with an asymptomatic patient from the cardiologic point of view.

On the 15th day of symptoms, due to clinical and body temperature worsening, he sought the emergency care department of a local hospital, where he was treated by an infectologist. At this point, a detailed clinical history was obtained. He had sustained high fever, above $39^{\circ} \mathrm{C}$, which did not improve with antipyretics used for the last five days (dipyrone $4 \mathrm{~g} /$ day and acetaminophen $3 \mathrm{~g} /$ day), accompanied by fetid and profuse night sweats (approximately four changes of clothing a night); asthenia; myalgia, especially in the dorsal region; mood alterations. The patient was a veterinarian who 
TABLE 1: Laboratory assessment.

\begin{tabular}{|c|c|c|c|}
\hline Variable/(unit)/(reference values) & Before symptom onset & Symptom onset & After 15 days \\
\hline Hemoglobin (g/dL) () & 13.6 & 12.6 & \\
\hline Hematocrit (\%) (37-47) & 40.5 & 39.5 & \\
\hline Leukocytes (per $\left.\mathrm{mm}^{3}\right)(4,000-10,000)$ & 4.500 & 4.300 & \\
\hline Neutrophils (per $\left.\mathrm{mm}^{3}\right)(2,000-8,000)$ & 2.520 & 1.720 & \\
\hline Lymphocytes (per $\left.\mathrm{mm}^{3}\right)(1,000-4,000)$ & 1.170 & 1.935 & \\
\hline Platelets $\left(\right.$ per $\left.\mathrm{mm}^{3}\right)\left(150-400,10^{3}\right)$ & 185.000 & 181.000 & \\
\hline Alanine aminotransferase $(\mathrm{U} / \mathrm{L})(<59)$ & 102 & & 95 \\
\hline Aspartate aminotransferase $(\mathrm{U} / \mathrm{L})(<72)$ & 51 & & 50 \\
\hline Gamma-glutamyltransferase (U/L) $(<73)$ & 162 & 226 & \\
\hline Lactic dehydrogenase (U/L) (313-618) & 660 & 828 & \\
\hline Chorionic gonadotropin $(\mathrm{mIU} / \mathrm{mL})(<5)$ & $<1$ & & \\
\hline Alpha-fetoprotein $(\mathrm{IU} / \mathrm{mL})(<5)$ & & 1.7 & \\
\hline Erythrocyte sedimentation rate $(\mathrm{mm})(<15)$ & & 15 & \\
\hline HIV I/II antibodies & & & Nonreagent \\
\hline Epstein Barr antibodies IgM $(\mathrm{U} / \mathrm{mL})(<20)$ & & & 10 \\
\hline Epstein Barr antibodies IgG $(\mathrm{U} / \mathrm{mL})(<20)$ & & & 288 \\
\hline Cytomegalovirus antibodies IgM & & & Nonreagent \\
\hline Cytomegalovirus antibodies IgG & & & Reagent \\
\hline Toxoplasmosis antibodies IgM & & & Nonreagent \\
\hline Toxoplasmosis antibodies IgG & & & Nonreagent \\
\hline Hepatitis B-surface antigen & & & Negative \\
\hline Hepatitis $\mathrm{C}$ - anti HCV antibodies & & & Nonreagent \\
\hline Antinuclear antibody & & & $1 / 80$ \\
\hline Rheumatoid factor $(\mathrm{IU} / \mathrm{mL})(<14)$ & & & 14.45 \\
\hline
\end{tabular}

had been exposed to the premature delivery of bovines in the past six months. He denied intake of dairy products at home.

Considering the symptoms and the epidemiology of exposure to cattle, the hypothesis of a brucellosis diagnosis was raised. Laboratory assessment was carried out immediately. The patient was released to undergo home observation and to be reevaluated within 48 hours. In this assessment, the serology screening test (Rose Bengal test) for Brucella sp. IgM was positive, while the IgG fraction was negative. The patient had three blood cultures in progress. Empirical antimicrobial therapy was initiated for brucellosis with doxycycline (dose = $200 \mathrm{mg}$ day) and rifampicin (dose $=900 \mathrm{mg} /$ day $)$.

He was maintained on outpatient assessment and reassessed on $07 / 22 / 12$, when blood culture results were released, showing to be positive for Gram-negative bacilli. New serologic tests for brucellosis were requested. The IgM fraction remained positive, while there was seroconversion from negative to positive IgG antibody to brucellosis. Treatment was maintained with the above-mentioned doses.

Other general laboratory tests showed slight increase in transaminases and positive antinuclear antibodies (speckled pattern). For other test results, see Table 1.

The patient was asymptomatic after 10 days of treatment. On reassessment after 30 days, he showed improved overall health status with the presence of mild night sweats, increased appetite and return to work activities. He had been afebrile for more than three weeks. Treatment was maintained at the above-mentioned doses and was completed in 42 days.
After 60 days of symptom onset, the patient was asymptomatic and afebrile. He was discharged and received instructions in case of symptom recurrence.

\section{Discussion}

In spite of its worldwide distribution, the fact of being considered endemic in some locations [2], and because of its broad spectrum of clinical manifestations - therefore having many differential diagnoses, both infectious and noninfectious [8], brucellosis remains underdiagnosed and underreported [9].

Among the wide spectrum of clinical manifestations [4], cardiovascular manifestations, such as endocarditis, myocarditis, and pericarditis are rare $[2,10]$. Of these, endocarditis is the most common, and the aortic valve is the most often affected site [10]. Endocarditis is also the most serious manifestation, contributing with more than $5 \%$ of the total rate of mortality by brucellosis [6]. When it occurs, the onset of myocarditis and pericarditis together is common, but its isolated appearance is extremely rare $[2,11]$.

After carrying out a search on MEDLINE database using the words "pericarditis" and "brucellosis," 18 references were identified, dating from 1982 to 2011 . Of these, only five papers contained reports of infections in humans with information in English on pericarditis associated with brucellosis, with the last cases having been reported in 2011 .

In a series of recent cases from a hospital located in Turkey, where the clinical manifestations were reviewed in 
240 patients diagnosed with brucellosis, cardiac manifestations suggestive of pericarditis were identified in two cases $(0.8 \%)$ [7]. In another series of cases including several hospitals, also located in Turkey, 1028 cases of brucellosis were retrospectively analyzed. Cardiac involvement was observed in seven patients (0.7\%), all diagnosed with endocarditis [1].

The first two cases published in English language in 1985 reported the finding of isolated pericarditis and important pericardial effusion as the main clinical manifestation, both progressing to cardiac tamponade [12], different from what occurred in this reported case, where pericarditis was asymptomatic and the main clinical manifestations were fever and involvement of the general health status.

On the other hand, and more similar to the present report, is a case reported in 2011, where a large-volume pericardial effusion was found at the transthoracic and transesophageal echocardiographies, which were performed to investigate endocarditis, as the patient had a systolic murmur on auscultation, although he was asymptomatic [10]. Our patient also underwent transthoracic echocardiography to rule out endocarditis, as part of investigation for fever of unknown origin. The possibility of endocarditis was excluded in our case, in spite of positive blood cultures, due to a combination of factors such as the absence of vegetations on echocardiography, the absence of a new heart murmur and a good clinical outcome after starting antibiotic therapy.

We chose not to perform a pericardiocentesis, as the patient was asymptomatic from the cardiovascular point of view and due to the size of the pericardial effusion.

The fact that the IgM serology for Brucella sp. was positive and there was seroconversion of IgG serology for Brucella sp., together with good clinical outcome after starting the antibiotic therapy corroborated the diagnosis.

Although the antibiotic regimen of choice for treatment of brucellosis is tetracycline plus streptomycin [5], the latter antibiotic is not released for treatment of brucellosis in Brazil. The regimen with doxycycline plus rifampicin is suited for oral treatment, when excluded skeletal complications such as discitis, meningitis, and endocarditis [5].

Considering that our patient was stable without requiring hospitalization, and showed no bone complications, meningitis, or endocarditis, we choose to use rifampicin plus doxycycline.

\section{Conclusion}

Cardiac manifestations are uncommon in the course of brucellosis, with the most common being endocarditis. Pericardial effusion may occur even as an isolated cardiovascular symptom and may manifest both symptomatically and asymptomatically. In areas where brucellosis is endemic, it is important to consider the fact that pericarditis can occur without symptoms due to the possibility of pericardial tamponade and sudden death. Furthermore, it is important to consider brucellosis as a differential diagnosis in cases of acute pericarditis.

\section{Conflict of Interests}

The authors declare that they have no conflict of interests.

\section{References}

[1] T. Buzgan, M. K. Karahocagil, H. Irmak et al., "Clinical manifestations and complications in 1028 cases of brucellosis: a retrospective evaluation and review of the literature," International Journal of Infectious Diseases, vol. 14, no. 6, pp. e469-e478, 2010.

[2] G. Pappas, N. Akritidis, M. Bosilkovski, and E. Tsianos, "Medical progress Brucellosis," New England Journal of Medicine, vol. 352, no. 22, pp. 2325-2367, 2005.

[3] G. Pappas, P. Papadimitriou, N. Akritidis, L. Christou, and E. V. Tsianos, "The new global map of human brucellosis," Lancet Infectious Diseases, vol. 6, no. 2, pp. 91-99, 2006.

[4] B. G. Mantur, M. S. Biradar, R. C. Bidri et al., "Protean clinical manifestations and diagnostic challenges of human brucellosis in adults: 16 years' experience in an endemic area," Journal of Medical Microbiology, vol. 55, no. 7, pp. 897-903, 2006.

[5] E. J. Young, "Brucella species," in Principles and Practice of Infectious Diseases, G. L. Mandell, J. E. Bennett, and R. Dolin, Eds., Churchill Livingstone, Philadelphia, Pa, USA, 2005.

[6] M. P. Franco, M. Mulder, R. H. Gilman, and H. L. Smits, "Human brucellosis," Lancet Infectious Diseases, vol. 7, no. 12, pp. 775-786, 2007.

[7] C. Ataman Hatipoglu, A. Yetkin, G. T. Ertem, and N. Tulek, "Unusual clinical presentations of brucellosis," Scandinavian Journal of Infectious Diseases, vol. 36, no. 9, pp. 694-697, 2004.

[8] P. Andriopoulos, M. Tsironi, S. Deftereos, A. Aessopos, and G. Assimakopoulos, "Acute brucellosis: presentation, diagnosis, and treatment of 144 cases," International Journal of Infectious Diseases, vol. 11, no. 1, pp. 52-57, 2007.

[9] J. Godfroid, A. Cloeckaert, J. P. Liautard et al., "From the discovery of the Malta fever's agent to the discovery of a marine mammal reservoir, brucellosis has continuously been a reemerging zoonosis," Veterinary Research, vol. 36, no. 3, pp. 313-326, 2005.

[10] N. K. Gatselis, K. P. Makaritsis, I. Gabranis, A. Stefos, K. Karanikas, and G. N. Dalekos, "Unusual cardiovascular complications of brucellosis presenting in two men: two case reports and a review of the literature," Journal of Medical Case Reports, vol. 5, article 22, 2011.

[11] J. D. Colmenero, J. M. Reguera, F. Martos et al., "Complications associated with Brucella melitensis infection: a study of 530 cases," Medicine, vol. 75, no. 4, pp. 195-211, 1996.

[12] M. C. Ugartemendía, A. Curós-Abadal, M. Pujol-Rakosnik, R. Pujadas-Capmany, E. Escrivá-Montserrat, and J. Jané-Pesquer, "Brucella melitensis pericarditis," American Heart Journal, vol. 109, no. 5, part 1, p. 1108, 1985. 


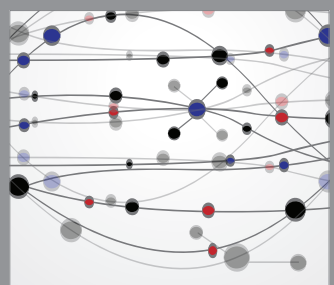

The Scientific World Journal
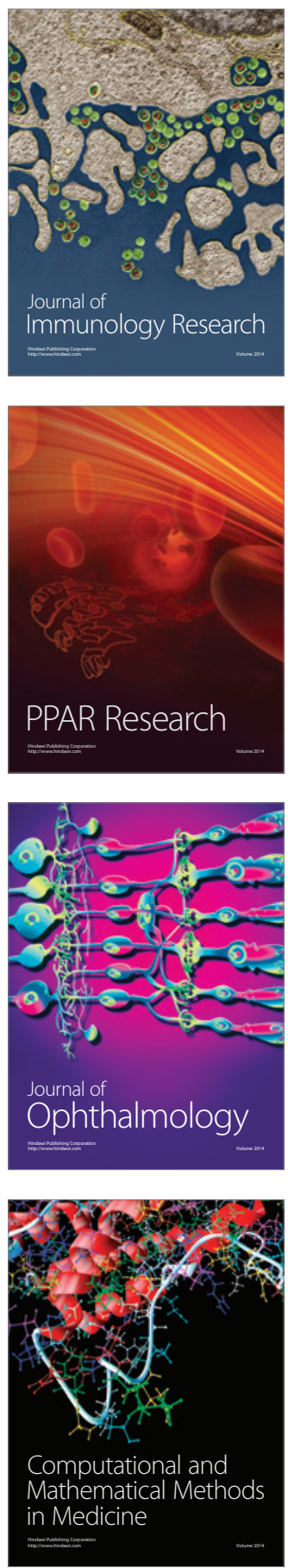

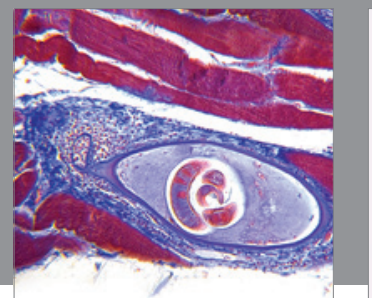

Gastroenterology

Research and Practice
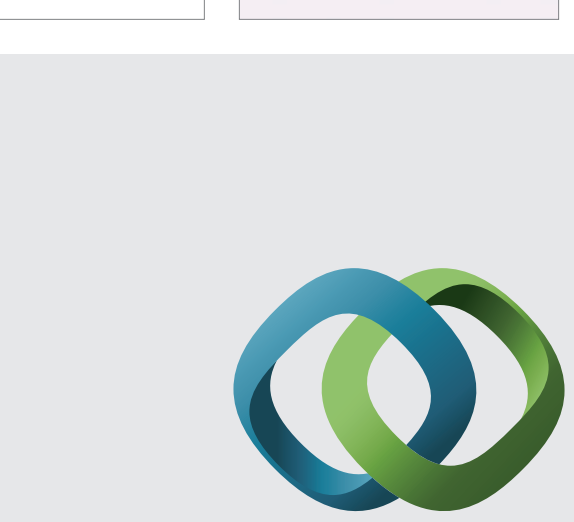

\section{Hindawi}

Submit your manuscripts at

http://www.hindawi.com
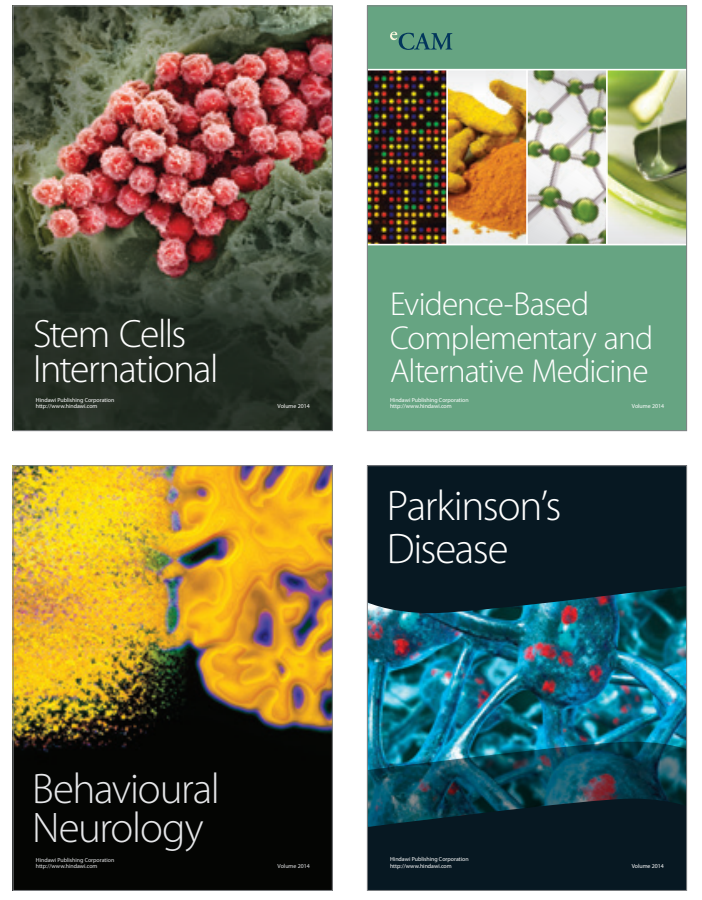
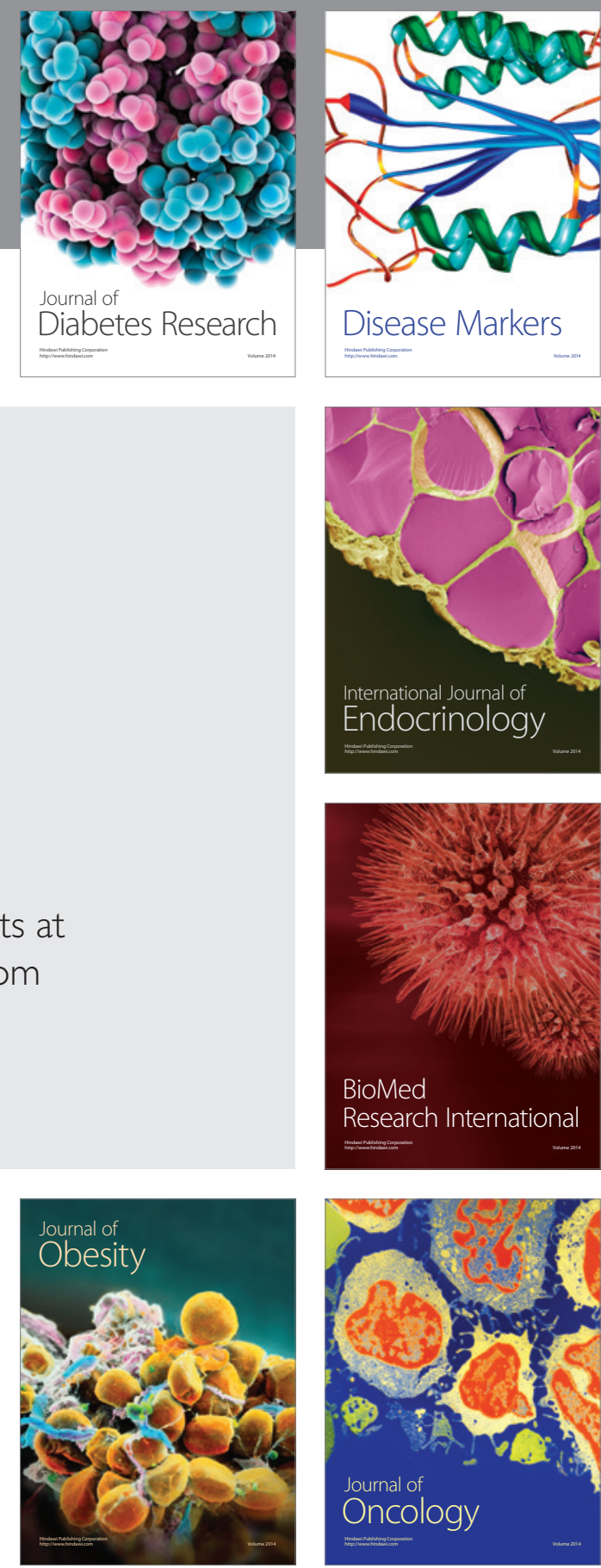

Disease Markers
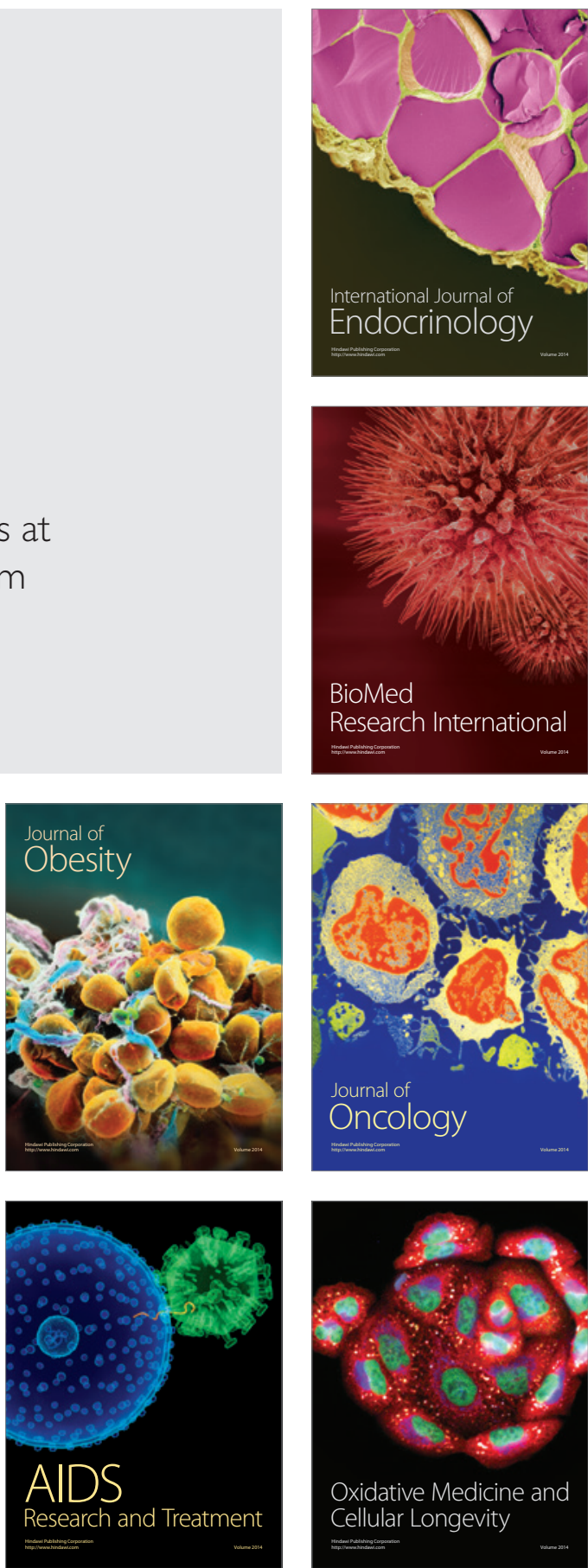